\title{
Update on monitoring of magnetic and electromagnetic tectonic signals in Central Italy
}

\author{
Domenico Di Mauro, Stefania Lepidi, Manuele Di Persio, Antonio Meloni and Paolo Palangio \\ Istituto Nazionale di Geofisica e Vulcanologia, Roma, Italy
}

\begin{abstract}
A network of three absolute magnetometer stations and the geomagnetic observatory of L'Aquila $\left(42^{\circ} 23 \mathrm{~N}\right.$, $13^{\circ} 19 \mathrm{E}$ ) monitors possible seismo- or tectonomagnetic effects in Central Italy, using L'Aquila Observatory as a reference for differentiation. A system of two VLF search coil wide-band antennas, working in two different frequency bands, at the L'Aquila Observatory, monitors possible electromagnetic effects related to seismic events occurring in Central Italy. Absolute magnetic field observations and VLF signals have been collected for several years. In particular the tectono-magnetic network started its operations in 1989. In this paper we report on the time variation of above mentioned data for the most recent years 2002 and 2003, also in connection with older measurements time series; we also report on seismic activity recorded in this area by the national seismic network. In the above mentioned time interval, no strong earthquake activity was recorded, and at the same time no clear evidence for magnetic or electromagnetic signals related to seismic events was found.
\end{abstract}

Key words tectonomagnetism - seismomagnetic network - wavelet - seismic activity - VLF - Central Italy

\section{Introduction}

Among various ground-based methods for monitoring the different kind of electromagnetic signals associated with the tectonic activity manifestation, like earthquakes or volcanic eruptions, the measurements of magnetic anomalies, in the frame of the so-called «tectonomagnetism», and of electromagnetic field emissions in ULF and VLF frequency bands, have been extensively used and described in recent papers (Johnston and Parrot, 1997; Meloni et al., 2001; Hayakawa and Molchanov, 2002).

Mailing address: Dr. Domenico Di Mauro, Istituto Nazionale di Geofisica e Vulcanologia, Via di Vigna Murata 605, 00143 Roma, Italy; e-mail: dimauro@ingv.it
Mechanisms for generating magnetic field changes associated with tectonic and volcanic activity are generally attributed to piezomagnetic and electrokinetic effects, assuming that rocks change their magnetization under mechanical or thermal stress and under variation of concentration gradients of electrolytes and flow of pore fluids in fractured rocks. All these mechanisms are strongly supported by laboratory tests, and at present, variations of a few nT have been claimed by observers, both in the case of earthquake and volcanic events (see for example Johnston, 1997; Del Negro and Currenti, 2003). Electromagnetic field emissions at low frequencies can be generally associated with piezoelectric effects or charge redistributions. At higher frequencies (from VLF on) the interaction and coupling of gravity waves induced by geochemical factors with the ionosphere and magnetosphere, could generate electromagnetic anomalous emissions; these disturbances can appear for a few days before and after an earthquake (Rozhnoi et al., 2004 and references therein). 


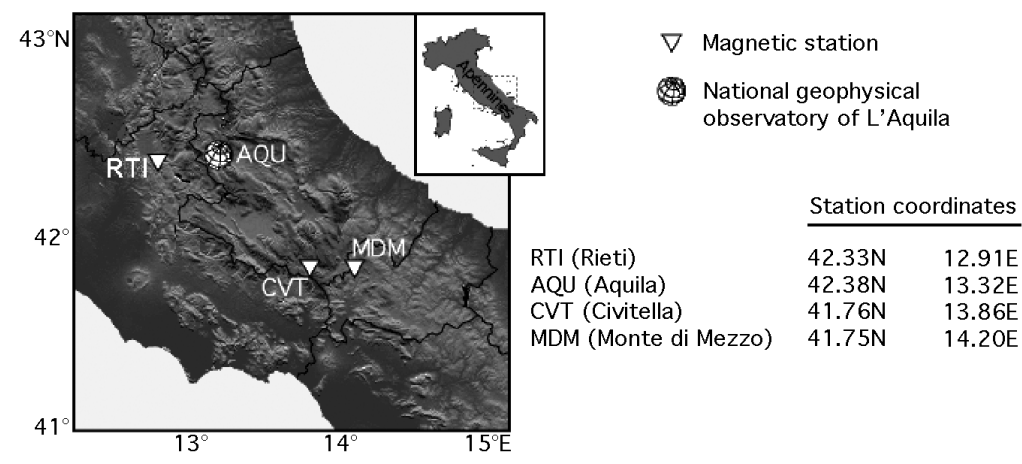

Fig. 1. Map of target area showing the location and geographic coordinates of magnetometer stations MDM, CVT, RTI and the national geomagnetic observatory at L'Aquila.

Following previous papers concerning tectonomagnetic and VLF observations in Central Italy, this paper shows the whole dataset which covers about fourteen years of recordings for tectonomagnetic observation with a particular attention to 2002 and 2003. Previous reports can be found in Meloni et al. (2001, 2004). We report on the absolute magnetic field variations by means of a network of magnetometer stations which started its operation in 1989. Data are differentiated with respect to the Italian magnetic observatory of L'Aquila $\left(42^{\circ} 23 \mathrm{~N}, 13^{\circ} 19 \mathrm{E}\right)$, used as a reference to eliminate non local tectonomagnetic fields on the dataset. In this latter location, a system of two VLF search coil wide-band antennas, each working in two different frequency bands, monitored the electromagnetic emissions, in the search for relations to seismic events in the area. Absolute magnetic field observations and VLF signals have been collected for several years, with some data gaps due to instrumental failures.

We also report on seismic activity recorded in this area by the national seismic network, for the above mentioned time interval and show some tentative correlation between magnetic signals and local and regional seismic activities.

\section{The network and instrumentation}

In an area of $100 \times 80 \mathrm{~km}^{2}$ extending between latitudes $41^{\circ}$ and $43^{\circ} \mathrm{N}$ and longitudes 12 and $15^{\circ}$, a network of four magnetometer stations is in operation, including the national geomagnetic observatory of L'Aquila, located in this area (fig. 1). The area is seismically active with a moderate-intense seismicity whose distribution, for this sector of Italy, follows approximately the NW-SE Apennines Mountain Chain direction. The experimental equipment characteristics, as well as more detailed information, can be found in mentioned papers (Meloni et al., 2001, 2004).

Each station is equipped with a proton procession magnetometer recording the total magnetic field intensity with a sampling rate of 15 min. Measured values are averaged and differentiated with respect to L'Aquila geomagnetic observatory data to filter out contributions from external sources and long term variations of the Earth's magnetic field of internal origin.

VLF signals along the E-W and S-N directions are monitored at L'Aquila Observatory by means of two search coil wide band antennas operating in the range $15-40 \mathrm{kHz}$. The system measures the signals RMS integrated over two frequency bands, centered at 20 and $40 \mathrm{kHz}$ respectively. Radiometer sensitivity is $200 \mathrm{fT}$ at $40 \mathrm{~Hz}$ and data are sampled with a 16 bit resolution, at $1 \mathrm{~Hz}$ frequency and averaged at $1 \mathrm{~min}$. The observatory area is characterized by a low level of artificial electromagnetic noise of local origin; in the frequency band of interest, radio signals emitted from several radio stations are recorded. 


\section{Data set and data analysis}

\subsection{Magnetic observations}

Time variations of the Earth's magnetic field have been collected since late 1989 in three sites in Central Italy, as an integration for tectonomagnetic studies to the institutional recordings performed at the national geomagnetic observatory of L'Aquila. The whole data set is shown in fig. 2 where sampled data are averaged daily. Station names and their abbreviations are given in fig. 1. The high frequency behaviour in each curve is caused by strong planetary magnetic activity, as modulated by the solar cycles, and the minimum of solar activity is indicated by a grey band.

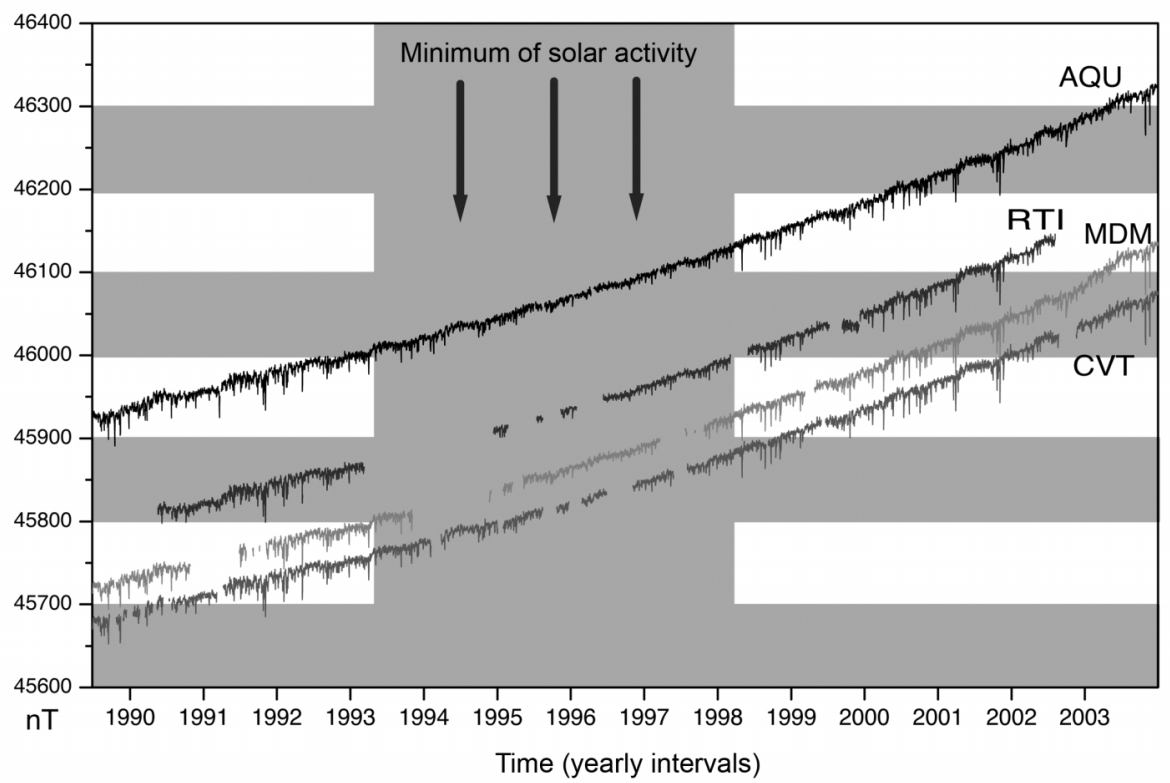

Fig. 2. Time variations of total magnetic field $F$ (daily averages) at L'Aquila (AQU) and at tectonomagnetic stations (CVT, MDM, RTI) from July 1989 to 2003.

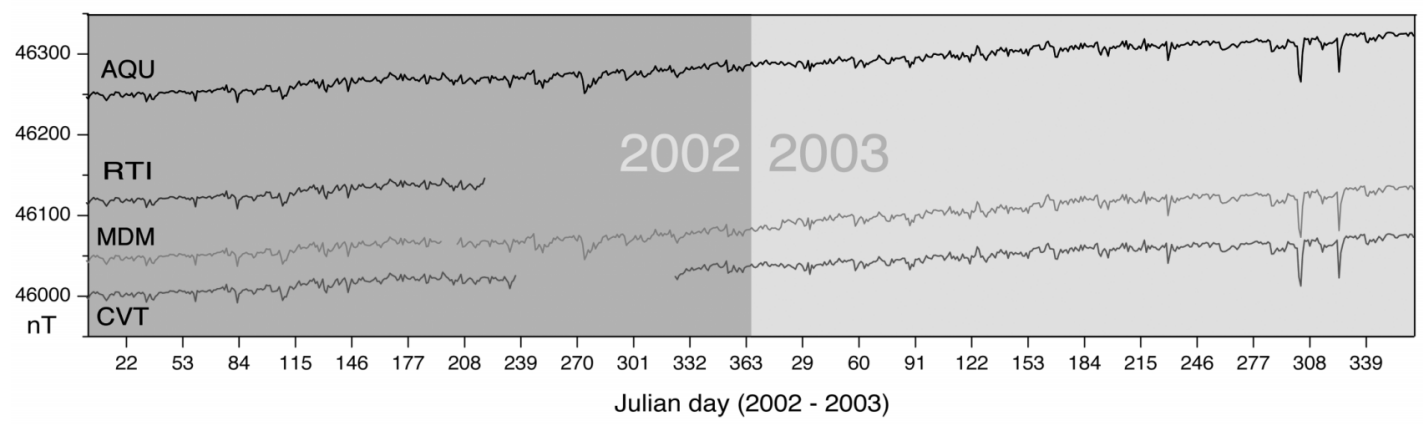

Fig. 3. Enlargement of time variations of total magnetic field $F$ (daily averages) at L'Aquila (AQU) and at tectonomagnetic stations (CVT, MDM, RTI) for the years 2002 and 2003. 
The last two years 2002 and 2003 of the presented data set are shown on a wider time window for a better visualization in fig. 3 .

The subtraction procedure between station pairs removes the inductive contributions due to the external electric currents in the ionosphere and magnetosphere. It also eliminates the long term contribution (large part of the non-uniform secular variation) of internal origin, due to the Earth's core electric currents. The remnant part is related to local changes in crustal magnetization, and possibly related to seismic and tectono-magnetic effects. Daily averages of differences between L'Aquila geomagnetic observatory and the stations CVT, MDM and RTI for 2002 and 2003 are shown in fig. 4. Vertical lines indicate the occurrence of the most intense earthquakes which occurred in the study area $\left(M_{d}>=4.0\right)$. Vertical grey bands indicate the occurrences of the Molise seismic sequence at the end of October 2002 and the Adriatic sea sequence of MarchApril 2003; see also table I for the full list.
Although some moderate seismicity and three $M>5$ events were recorded in the area in 2002 and 2003, no clear evidence for seismomagnetic effects was found. Unfortunately due to a technical problem the RTI station has interrupted its data acquisition on late July 2002.

Two rapid decreases of about $10 \mathrm{nT}$ and about 5 nT were recorded in the difference AQU-MDM at the beginning and at mid 2003, respectively. Such decreases are persistent for the rest of the year and are not due, to our knowledge, to technical failures or man-made interferences; these events do not have immediate time relation to recorded earthquakes and, at this time, no reasonable explanation was found; they need more investigation.

Wavelet analysis is a powerful tool to decompose a signal into its frequency content, and then to study each content with a resolution matched to its scale. It has advantages over traditional Fourier methods in analyzing physical situations where the signal contains discontinuities and spikes (Da-

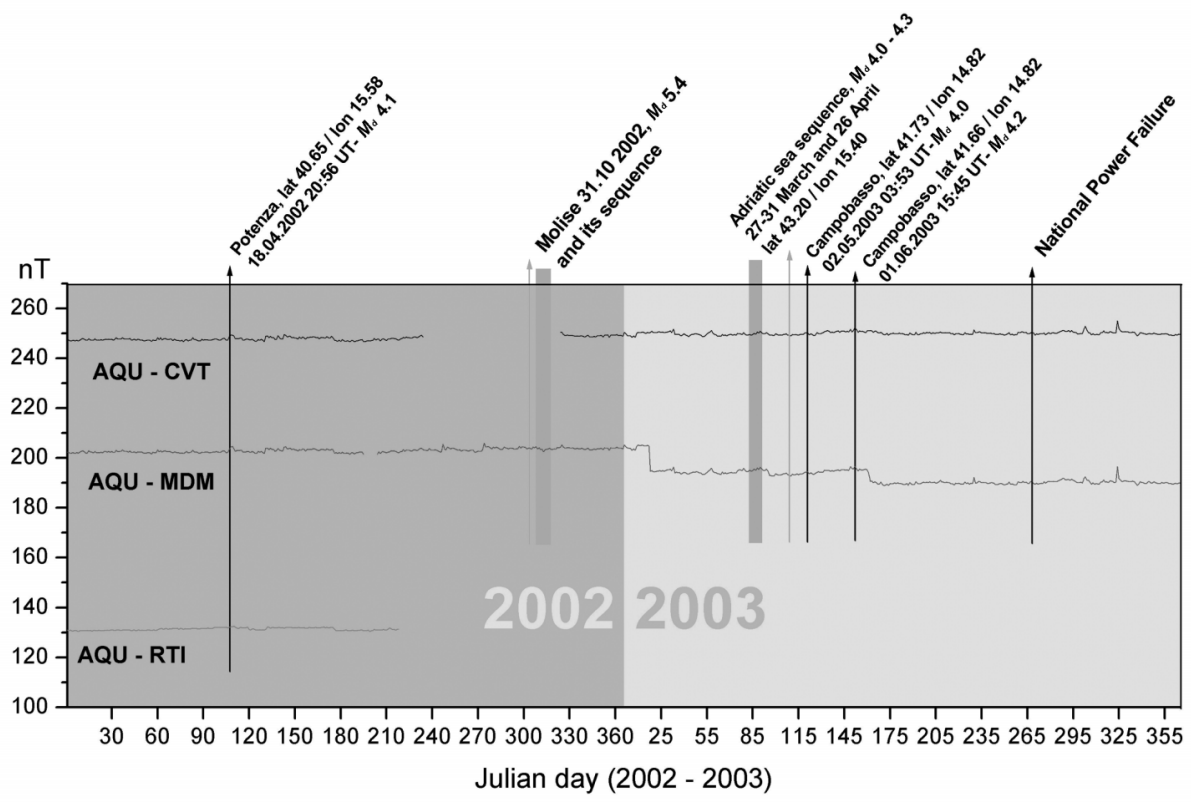

Fig. 4. Daily differences (in nT) between couples of stations and main seismic events occurred in 2002 and 2003 in an area extending $41^{\circ}$ and $43^{\circ}$ in latitude and $12^{\circ}$ and $15^{\circ}$ in longitude. Vertical bands indicate the Molise earthquake $(M=5.4)$ and its sequence occurred at the end of October 2002 and the Adriatic Sea sequence of MarchApril 2003. National power blackout event occurred on September 28, 2003 at 03:01 is also reported on plot. 
Table I. List and geographic location of earthquakes with $M \geq 4.0$ occurred in the target area of Central Italy during 2002 and 2003. Source: INGV (2004).

\begin{tabular}{lcccl}
\hline \hline \multicolumn{1}{c}{ Date and time } & Magnitude & Lat & Long & Marks \\
\hline 01 June 2003 15:45:17 & 4.2 & 41.68 & 14.80 & \\
01 June 2003 01:46:00 & 4.0 & 43.19 & 15.43 & Campobasso earthquake \\
05 May 2003 06:16:00 & 4.2 & 43.13 & 15.38 & \\
02 May 2003 03:53:07 & 4.0 & 41.73 & 14.82 & Campobasso earthquake \\
26 April 2003 08:21:00 & 4.1 & 43.20 & 15.45 & \\
31 March 2003 19:36:28 & 4.3 & 43.09 & 15.38 & \\
30 March 2003 11:10:00 & 4.4 & 43.10 & 15.40 & \\
30 March 2003 00:56:22 & 4.0 & 43.19 & 15.43 & \\
29 March 2003 17:42:00 & 5.4 & 43.13 & 15.41 & \\
27 March 2003 16:14:00 & 4.0 & 43.20 & 15.49 & \\
27 March 2003 16:10:00 & 4.7 & 43.20 & 15.49 & Adriatic sea sequence \\
12 November 2002 09:27:48 & 4.2 & 41.68 & 14.79 & \\
04 November 2002 03:26:00 & 4.1 & 41.73 & 14.80 & \\
04 November 2002 00:35:00 & 4.2 & 41.67 & 14.82 & \\
01 November 2002 17:21:00 & 4.3 & 41.71 & 14.82 & \\
01 November 2002 15:20:00 & 4.1 & 41.74 & 14.85 & \\
01 November 2002 15:08:00 & 5.3 & 41.69 & 14.83 & \\
31 October 2002 10:32:00 & 5.4 & 41.76 & 14.94 & Molise earthquake and sequence \\
18 April 2002 20:56:00 & 4.1 & 40.65 & 15.58 & Potenza earthquake \\
\hline
\end{tabular}

ubechies, 1988; Meyer, 1993). In this study the valuable use of wavelets is twofold: to characterize the behaviour of each station and to compare how each station responds to rapid changes.

Examples of wavelet analysis applied to the differences AQU-MDM and AQU-RTI for the month of April 2002 when an earthquake occurred on April 18, 2002 at 20:56 (UT) at 40.65N and $15.58 \mathrm{E}$, are shown in fig. 5a,b. Despite outside the network limits such event occurred when the three stations were in operation. A multi-level decomposition by means of «db3» wavelet applied to the month of April 2002 data gives the approximation and the detail panels from the most similar to the recorded signal (approximation 1) to the less accurate (approximation 5). As expected, the signal decompositions of the differences for the closest stations of MDM and CVT (not shown) with respect to AQU are comparable; while AQU-RTI decomposition reveals an individual behaviour especially at lower approximations.
At approximation «a5» (left side of fig. 5a) of AQU-RTI we note the separation of two different domains at the earthquake occurrence. No similar behaviour can be seen on the panel related to AQU-MDM even if MDM is closer to the earthquake focus. Wavelet coefficients (upper right panel) represent the inner products of the signal and wavelet basis. They can be interpreted as the cross-correlation function which tell us the similarity between the signal an the wavelet basis.

Further applications of wavelet decomposition on data intervals containing the signal jumps previously mentioned was performed. For what concerns the largest jump of $\sim 10$ nT occurred in January 2003, a comparison of AQU-MDM and AQU-CVT is shown in fig. 6. In this case, decompositions of both differences help us to exclude that a jump could be hidden in signals from other stations where in fact no evidence of change occurred also by visual inspection. A relationship to any physical phenomena, as a source for that jump, needs further investigation. 

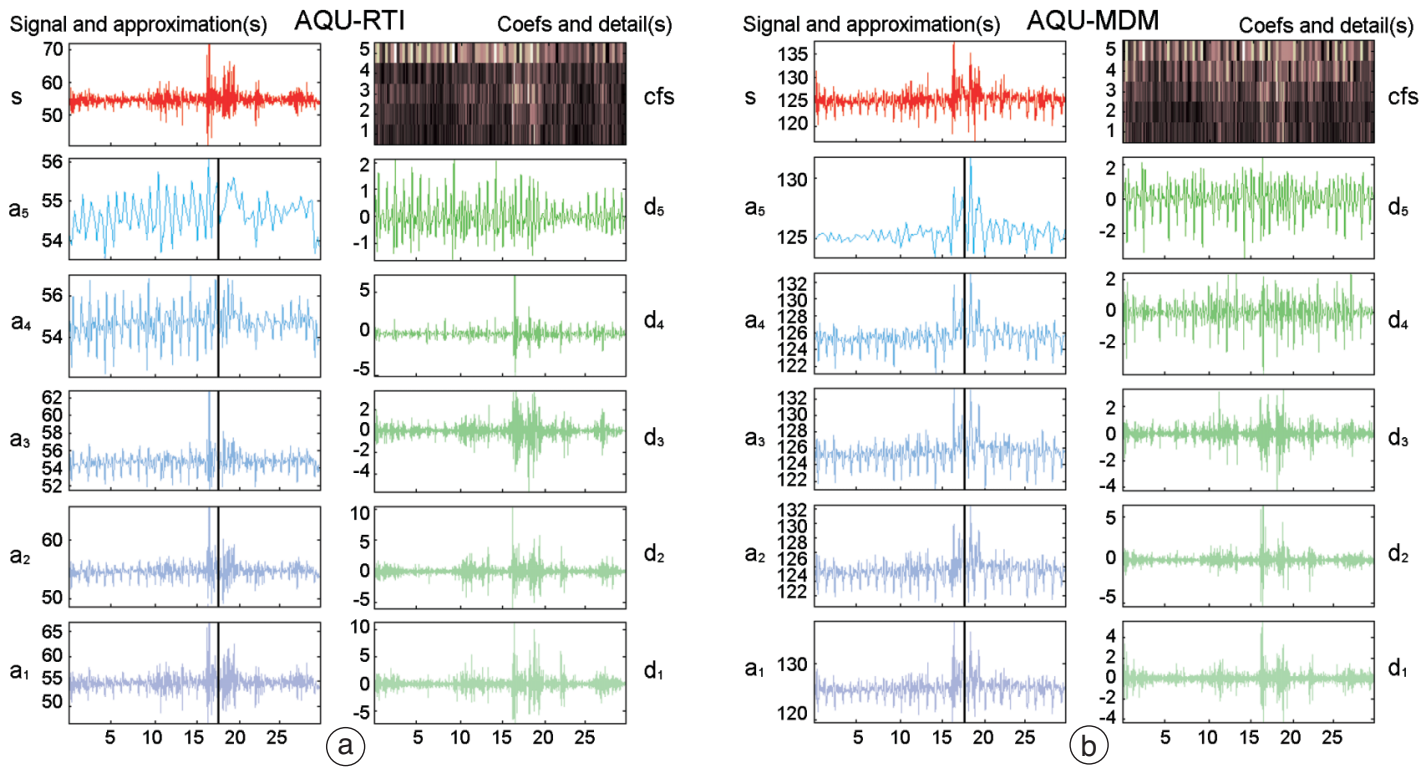

Fig. 5a,b. A 5-level decomposition of the differences a) AQU-RTI and b) AQU-MDM for the month of April 2002 by means of «db3» wavelet. On April 18th 2002 an earthquake occurred outside the area covered by the network (marked as vertical line in left panels). Signal and approximations from step1 to 5, and coefficients and details from step1 to 5 are shown in the left and right hand side, respectively (see text). In each approximation and detailed panels, the horizontal axis indicates time in days while in the vertical axis unit is nT.

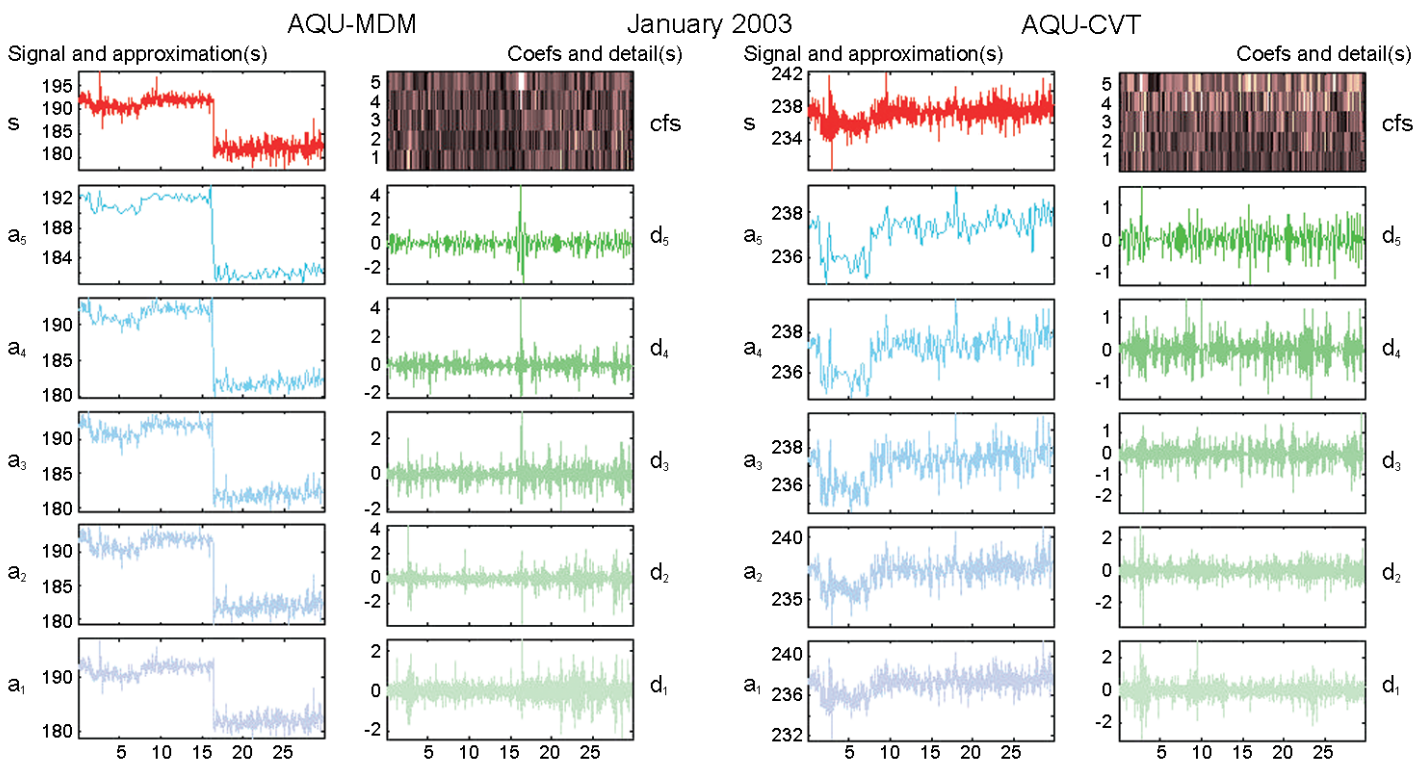

Fig. 6. A decomposition of the differences AQU-MDM and AQU-CVT for January 2003 by means of «db3» wavelet at level 5 applied on one month of data (January 2003). 
$20 \mathrm{kHz}$ E-W; March-September 2001
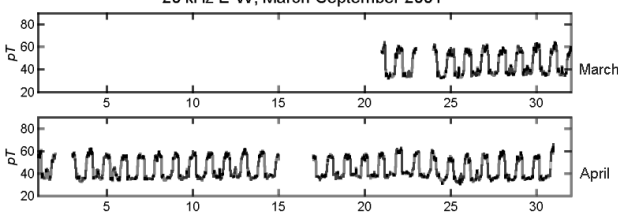

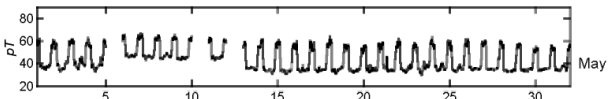
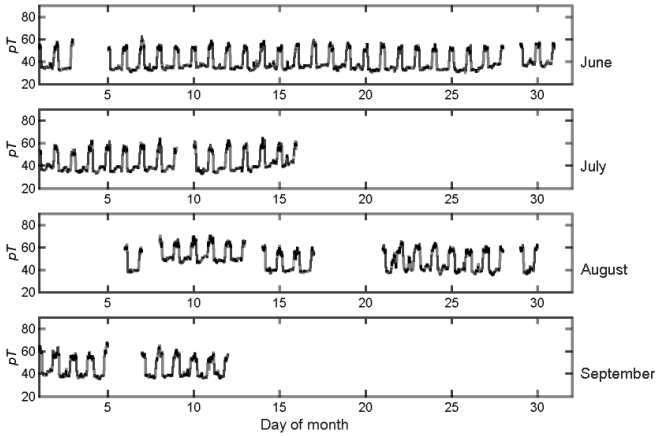

$40 \mathrm{kHz}$ E-W; March-September 2001

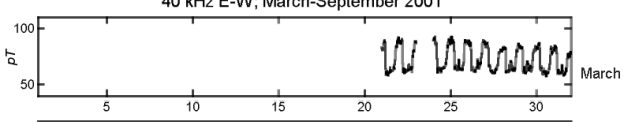

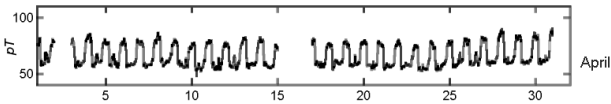

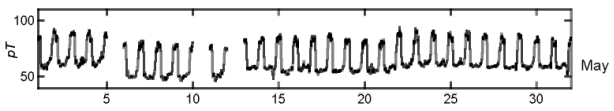

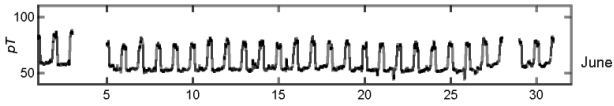
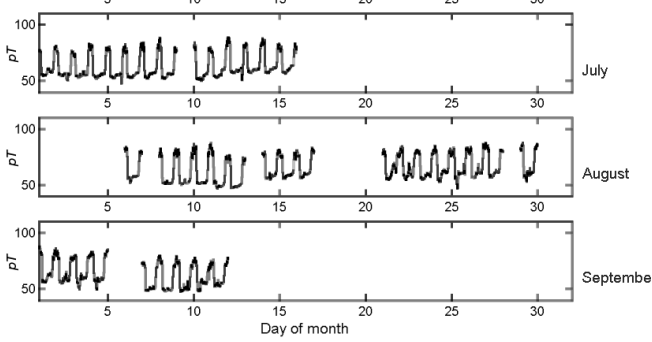

$20 \mathrm{kHz}$ E-W; August 2002 - March 2003

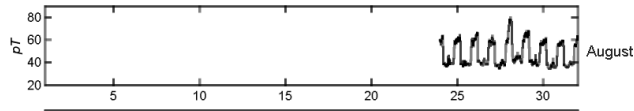

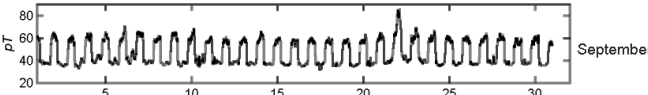

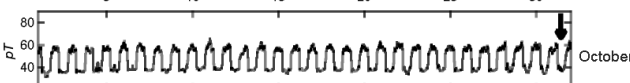
\begin{tabular}{cccccc}
1 & 1 & 1 & 1 & 1 & 1 \\
\hline 5 & 10 & 15 & 20 & 25 & 30
\end{tabular}

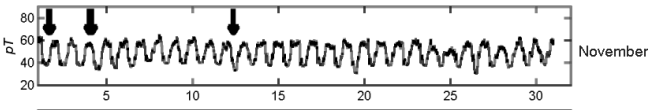

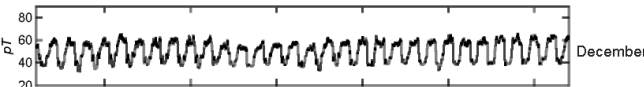
\begin{tabular}{rrrrrr}
20 & 1 & 1 & 1 & 1 & 1 \\
\hline 5 & 10 & 15 & 20 & 25 & 30
\end{tabular}

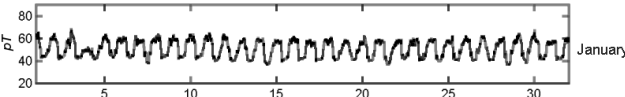

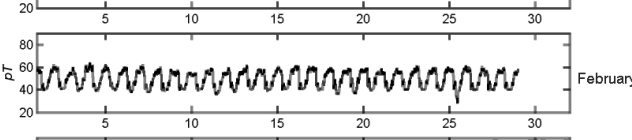

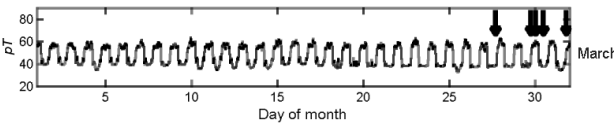

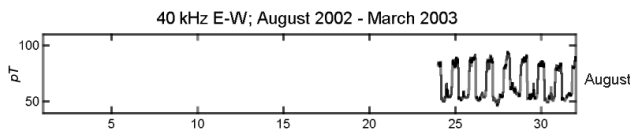

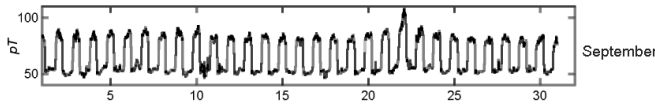

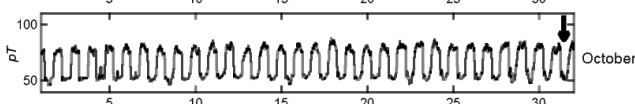
$\underbrace{100}_{50}$ $\underbrace{}_{5}$

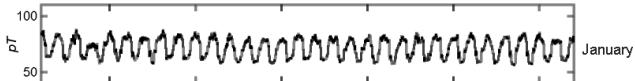
\begin{tabular}{cccccc}
1 & 1 & 1 & 1 & 1 \\
\hline
\end{tabular} $=\underbrace{100}_{5}$

Fig. 7. VLF $20 \mathrm{kHz}$ and $40 \mathrm{kHz}$ data (1 min averages) for the time periods March-September 2001 and September 2002-March 2003. The arrows indicate the earthquake $\left(M_{d}>=4\right)$ occurrence. 


\subsection{VLF observations}

The time variability of VLF electromagnetic signals, along E-W direction, for the periods March-September 2001 and August 2002March 2003, is shown in fig. 7. VLF data certainly show no significant anomalies in correspondence to the seismic events (indicated in the plots by the arrows). Moreover, in the whole analyzed time series, no anomalous enhance- ment of the signal comparable to those recorded in correspondence of the 1997 seismic events is present (Meloni et al., 2001).

In monitoring the ionospheric characteristics that could be related in some way to tectonomagnetic events, an important parameter is the difference between the daytime and nighttime VLF amplitude levels, which is independent of possible changes in the data offsets. In order to compute this parameter, we took as
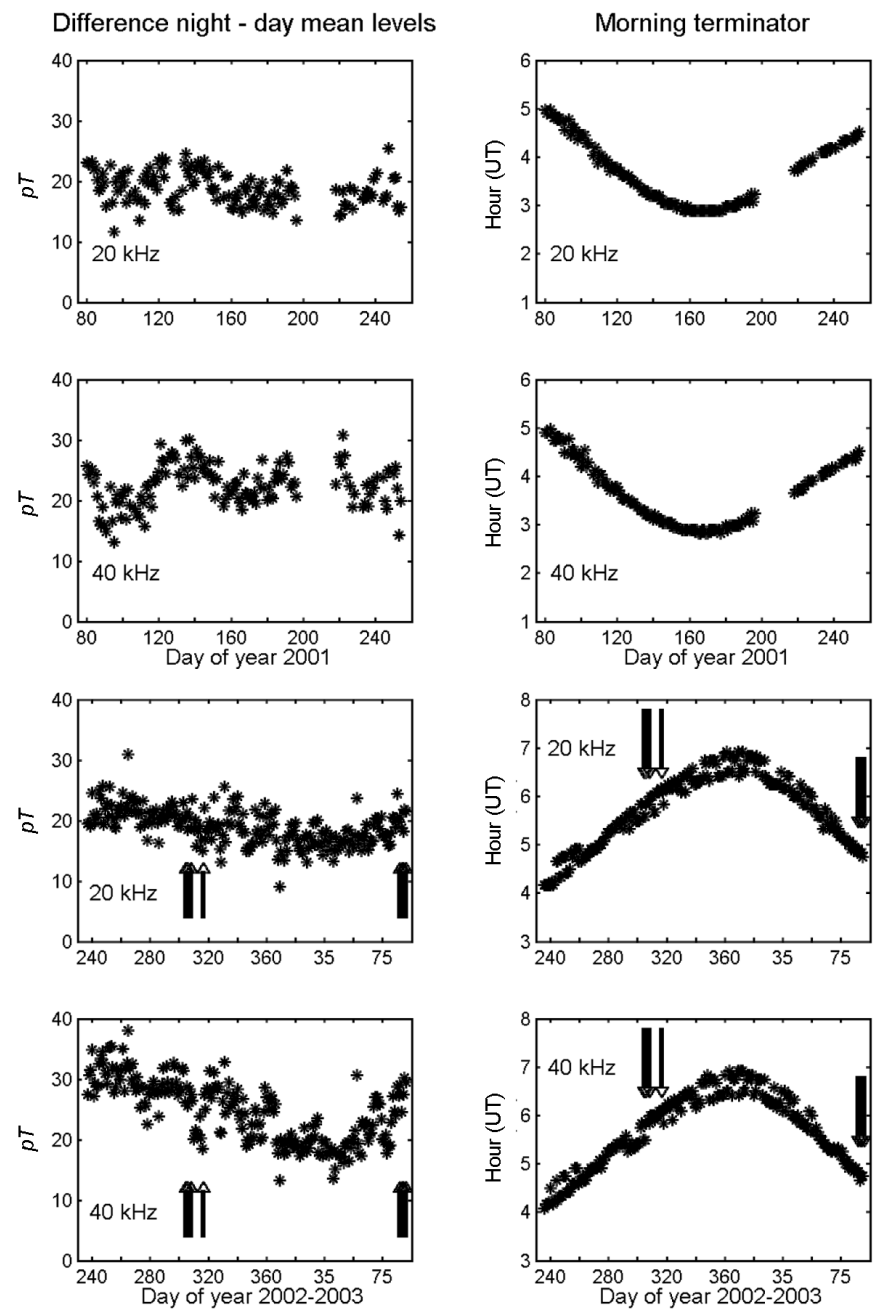

Fig. 8. Left panels: difference between the average nighttime (10-14 LT) and daytime (22-02 LT) VLF levels; right panels: morning terminator time. The arrows indicate the earthquakes $\left(M_{d}>=4\right)$ occurrence. 
daytime and nighttime levels the intervals 1014 LT and 22-02 LT, respectively. Results, shown in the left panels of fig. 8, prove that in the two analyzed time periods the difference between the daytime and nighttime VLF levels does not exhibit clear irregularities in correspondence to the seismic events (indicated in the plots by the arrows); the data rather show some evidence for a seasonal variation, more evident on the $40 \mathrm{kHz}$ signal.

Another physical quantity that provides information on ionospheric long-term changes is the so called Terminator Time TT, i.e. the time of the daily variation in the morning and evening. Previous studies have shown anomalous shifts of the TT which appear some days before large earthquakes and disappear a few days after them (Hayakawa et al., 1996a,b; Molchanov and Hayakawa, 1998; Rozhnoi et al., 2004).

We calculated the morning TT, defined as the time of the minimum in the differenced data, previously averaged at $5 \mathrm{~min}$ to remove high frequency variations and noise which gave spurious minima. The variation of the TT during the analyzed time periods is shown in the right panels of fig. 8. The expected seasonal variation of the TT, related to the change of the sunrise time shows clearly; it is also evident that during summer TT is better defined, while during winter its values are more scattered. Also in this case there is no clear correspondence between TT anomalies and reported seismic events.

\section{Conclusions}

Seismomagnetic and VLF data gathered in recent years were used to obtain a better knowledge of the tectonomagnetic and electromagnetic phenomena observed in this region. Data were also analyzed to evaluate if results could be used in the search of possible correlation to seismic activity. No evidence of crustal magnetic time variations in association to earthquake occurrence was clearly found. Wavelet analysis was applied to seismomagnetic signals in order to better characterize the behaviour of each station and to compare their response when the signal contains discontinuities and spikes.
Continuous VLF observation represents an important tool in the assessment of earthquake electromagnetic related phenomena even if VLF wave plots, here shown for 20 and $40 \mathrm{kHz}$ in their 1-min averages, do not reveal significant variations comparable to those recorded in the 1997 event (Meloni et al., 2001). Data achieved in subsequent years have shown that evident variations are not frequently recorded at L'Aquila and at the same time no other significant earthquake was recorded. We note, however, that the connection between VLF signals perturbations and earthquake occurrence was previously found for events with $M>5.5$. More data are needed to reach convincing conclusions but continuous monitoring of tectonomagnetic and VLF integrated averages time variations will be continued for their promising role in the assessment of earthquake related electromagnetic phenomena in this area of Central Italy.

\section{Acknowledgements}

The authors are grateful to the technical staff of the L'Aquila Observatory, in particular to F. Biasini and C. Gizzi, for practical support in the experimental activity.

\section{REFERENCES}

DAUBeChIES, I. (1988), Orthonormal bases of compactly supported wavelets, Comm. Pure Appl. Math., 41, 906-966.

Del Negro, C. and G. Currenti (2003): Volcanomagnetic signals associated with the 2001 flank eruption of Mt. Etna (Italy), Geophys. Res. Lett., 30 (7), 1357.

HayaKaWa, M. and O.A. Molchanov (Editors) (2002): Seismo-Electromagnetics: Lithosphere-Atmosphere-Ionosphere Coupling (Terra Scientific Publishing Co., Tokyo), pp. 477.

HayaKawa, M., O.A. Molchanov, T. Ondoh and E. KaWAY (1996a): The precursory signature effect of the Kobe earthquake in VLF subionospheric signal, J. Comm. Res. Lab., 43, 169-180.

Hayakawa, M., O.A. Molchanov, T. Ondoh and E. Kaway (1996b): Anomalies in the sub-ionospheric VLF signals for the 1995 Hyogo-ken Nanbu earthquake, $J$. Phys. Earth, 44, 413-418.

INGV (2004): Seismic Bulletin (Istituto Nazionale di Geofisica e Vulcanologia, Roma, Italy).

Johnston, M.J.S. (1997): Review of electrical and magnetic fields accompanying seismic and volcanic activity, Surv. Geophys., 18, 441-475. 
Johnston, M. and M. PARrot (1997): Electromagnetic effects of earthquakes and volcanoes, Phys. Earth Planet. Int., 105, 109-118.

Meloni, A., D. Patella, F. Vallianatos and B. Zolesi (Editors) (2001): Magnetic, electric and electromagnetic methods in seismology and volcanology, Chania Greece, Ann. Geofis., 44 (2), pp. 320.

Meloni, A., D. Di Mauro, S. Lepidi, G. Mele and P. PALANGIO (2004): Tectonomagnetic and VLF electromagnetic signals in Central Italy, Ann. Geophysics, 47 (1), 29-34
Meyer, Y. (1993): Wavelets: Algorithms and Applications (Society for Industrial and Applied Mathematics, Philadelphia), 13-31, 101-105.

Molchanov, O.A. and M. HayaKawa (1998): Subionospheric VLF signal perturbations possibly related to earthquakes, J. Geophys. Res., 103 (A8), 17489-17504.

RozhnoI, A., M.S. Solovieva, O.A. Molchanov and M. HaYAKawA (2004): Middle latitude LF (40 kHz) phase variations associated with earthquakes for quiet and disturbed geomagnetic conditions, Phys. Chem. Earth, 29, 589-598. 